\title{
Playing around the anaerobic threshold during COVID-19 pandemic: advantages and disadvantages of adding bouts of anaerobic work to aerobic activity in physical treatment of individuals with obesity
}

\author{
Alberto Salvadori ${ }^{1}$ - Paolo Fanari ${ }^{1} \cdot$ Paolo Marzullo ${ }^{2,3}$. Franco Codecasa ${ }^{1} \cdot$ Ilaria Tovaglieri $^{1} \cdot$ Mauro Cornacchia $^{1}$. \\ Ileana Terruzzi $i^{6,7}$. Anna Ferrulli ${ }^{6,7}$. Patrizia Palmulli ${ }^{1}$. Amelia Brunani ${ }^{4}$. Stefano Lanzi $i^{5}$ Livio Luzi $^{6,7}$ (D)
}

Received: 16 March 2021 / Accepted: 15 May 2021 / Published online: 28 May 2021

(c) The Author(s) 2021

\begin{abstract}
Introduction Obesity is a condition that generally limits work capacity and predisposes to a number of comorbidities and related diseases, the last being COVID-19 and its complications and sequelae. Physical exercise, together with diet, is a milestone in its management and rehabilitation, although there is still a debate on intensity and duration of training. Anaerobic threshold (AT) is a broad term often used either as ventilatory threshold or as lactate threshold, respectively, detected by respiratory ventilation and/or respiratory gases $\left(\mathrm{VCO}_{2}\right.$ and $\left.\mathrm{VO}_{2}\right)$, and by blood lactic acid.

Aims and methodology This review outlines the role of AT and of the different variations of growth hormone and catecholamine, in subjects with obesity vs normal weight individuals below and beyond AT, during a progressive increase in exercise training. We present a re-evaluation of the effects of physical activity on body mass and metabolism of individuals with obesity in light of potential benefits and pitfalls during COVID-19 pandemic. Comparison of a training program at moderate-intensity exercise $(<\mathrm{AT})$ with training performed at moderate intensity $(<\mathrm{AT})$ plus a final bout of high-intensity $(>\mathrm{AT})$ exercise at the end of the aerobic session will be discussed.

Results Based on our data and considerations, a tailored strategy for individuals with obesity concerning the most appropriate intensity of training in the context of rehabilitation is proposed, with special regard to potential benefits of work program above AT.

Conclusion Adding bouts of exercise above AT may improve lactic acid and $\mathrm{H}^{+}$disposal and improve growth hormone. Long-term aerobic exercise may improve leptin reduction. In this way, the propensity of subjects with obesity to encounter a serious prognosis of COVID-19 may be counteracted and the systemic and cardiorespiratory sequelae that may ensue after COVID-19, can be overcome. Individuals with serious comorbidities associated with obesity should avoid excessive exercise intensity.
\end{abstract}

Keywords Anaerobic threshold $\cdot$ High-intensity $\cdot$ Obesity $\cdot$ Physical exercise $\cdot$ Rehabilitation $\cdot$ covid-19

Managed by Antonio Secchi .

Livio Luzi

livio.luzi@unimi.it

1 Department of Pulmonary Rehabilitation, Istituto Auxologico Italiano IRCCS, Verbania (VB), Italy

2 Division of General Medicine, Ospedale S. Giuseppe, Istituto Auxologico Italiano, via Cadorna 90, 28824 Piancavallo Di Oggebbio (VB), Italy

3 Department of Translational Medicine, University of Piemonte Orientale, via Solaroli 17, 28100 Novara, Italy
4 Department of Rehabilitation Medicine, Istituto Auxologico Italiano IRCCS, Verbania (VB), Italy

5 Division of Angiology, Heart and Vessel Department, Lausanne University Hospital, Lausanne, Switzerland

6 Department of Endocrinology, Nutrition and Metabolic Diseases, IRCCS MultiMedica, Via Milanese 300, Sesto San Giovanni, Italy

7 Department of Biomedical Sciences for Health, University of Milan, Milan, Italy 


\section{Introduction}

\section{Obesity, exercise, and COVID-19}

In humans, both physiological and neuroendocrine factors act to control the amount of biological activity of white adipose tissue. In subjects with obesity, a reduced metabolic response to sympathetic nervous system (SNS) activity is known [1,2], with a blunted lipolytic action on visceral adiposity $[3,4]$. Several hormones including corticosteroids, growth hormone (GH), androgens and estrogens are less responsive to exercise in subjects with obesity [5,6]. All the above contribute to regulating lipid metabolism in response to exercise training. Yet, regular exercise is an important step, together with diet, in the management and rehabilitation strategies of individuals with obesity, due to its ability to modulate both components of the energy balance equation, namely energy intake and energy output [6]. Furthermore, we recently reported on the positive immunomodulatory effects of physical exercise protecting against COVID-19 [7]. Since obesity is at the same time a risk factor for the contagion by SARS- $\mathrm{CoV}_{2}$ and predisposes infected individuals to a worse prognosis [8], herein we present several considerations on the binomial obesity-exercise intensity and COVID-19.

In general, subjects with obesity have a decreased work capacity when compared to lean subjects, mostly due to an increased cardiac left ventricular mass and wall thickness with abnormalities in diastolic filling [9]. Moreover, they must overcome a decreased compliance in respiratory system [10]. Maximal sustainable work outputs in young subjects with obesity are similar to those of lean age-matched controls, but they attain the anaerobic threshold (AT) at significant lower outputs [11]. This means that endurance time at maximal effort is shorter, and therefore, that work capacity is lower [12]. Older subjects with obesity show a significant reduction in maximal sustainable work capacity and reach earlier AT, reflecting a more severe reduction in physical performance as compared to controls [13].

Furthermore, individuals with obesity have an altered dynamic of pulmonary ventilation, which is a potential cause of the worse clinical picture of COVID-19 [8]. During a progressive exercise, the delta increment of oxygen consumption is the same in subjects with obesity and in normal weight subjects. Nevertheless, obesity implies a constant significant greater absolute amount of oxygen at each correspondent external work output. This suggests that gross mechanical efficiency (Watts/VO $/ \mathrm{V}_{2}$ ) is lower in individuals with obesity despite similarities in net mechanical efficiency (Watts $/ \mathrm{VO}_{2}-\mathrm{VO}_{2}$ freewheeling) when compared to controls [11]. The underlying mechanism involves a higher peripheral oxygen uptake in individuals with obesity than in controls. This is partly due to a different pattern in circulating endothelin-1/NO concentrations, with a decrease in the former and a higher level of the latter in subjects with obesity [13]. Exercising above AT increases the capacity of both lean subjects and individuals with obesity, to remove lactic acid from the circulation. Hyperlactatemia and lactic acidosis are among the precipitating factors of multi-organ failure, the most dreadful complication of COVID-19 [8]. Therefore, upregulating lactic acid disposal capacity constitutes a defense against multi-organ failure mainly for subject with a low AT as individuals with obesity [8]. Furthermore, in a diagram representing AT plotted against body mass index (BMI) (or percent of ideal weight), a linear increase can be observed in normal subjects, while a linear decrease can be documented in subjects with obesity [14], suggesting that in individuals with obesity the decrease in AT is proportional to the increase in body mass.

\section{Anaerobic threshold, lactic acid, respiratory exchange ratio (RER)}

The intensity of physical work plays a role in the utilization of metabolic substrates. At low-intensity exercise $(<30 \%$ of $\mathrm{VO}_{2} \mathrm{max}$ ), lipids deriving from adipose tissue store are used predominantly; at moderate-intensity exercise (40-65\% $\mathrm{VO}_{2} \max$ ) significant amounts of fats from both adipose tissue and intramuscular stores are used; at high-intensity exercise ( $\left.>70 \% \mathrm{VO}_{2} \mathrm{max}\right)$, small amounts of fats are used, while glucose and glycogen become the predominant energy substrates [15].

AT indicates the $\mathrm{VO}_{2}$ level at which starts the anaerobic supplementation of the aerobic energy production. Both arterial lactate and lactate/pyruvate ratio increase for a given subject depending on his fitness and form of exercise [16]. It may be a useful index for evaluating daily life activity and in prescribing exercise regimen [17].

Methods using lactatemia and/or ventilation are reference methods to fix working intensities. They allow the detection of two lactic thresholds and two ventilator thresholds. The former threshold (lactic or ventilatory) is mainly useful for training programs in ill subjects, while the latter (lactic or ventilatory) is useful for training programs in healthy subjects and athletes [18]. Ventilatory threshold is used as an effort-independent physiological marker of the ability to perform submaximal, prolonged physical activity [19].

The aerobic energy system (below AT) produces the energy source adenosine triphosphate (ATP) by oxidative pathways, being configured for long-term steady work. The anaerobic alactic and lactic acid systems are less efficient than the aerobic system, producing quickly energy by nonoxidative pathways [20]. Their activation is short lasting, 
depending on the limited glycogen stores in human body and consequent to the accumulation of fatigue-related metabolites [e. g. $\mathrm{H}^{+} \mathrm{Pi}$ and extracellular $\mathrm{K}^{+}$[21]].

The most feared event complicating COVID-19 is multiorgan failure, which entails a failure of mitochondrial ATP production in many vital organs such as lungs but also heart, kidneys, gut and brain. COVID-19 in patients with obesity involves a higher mortality due to several factors among which the cellular respiratory chain failure predominates [22]. During multi-organ failure, lactic acid along with fatigue and stress-related metabolites (mainly $\mathrm{H}^{+}$) increases due to a switch toward anaerobic metabolism. To note that the switch takes place earlier in subjects with obesity (namely at lower workloads) and even at very low workloads (e.g., simply walking) in individuals with stage III obesity.

The Respiratory Exchange Ratio (RER) is the ratio between $\mathrm{CO}_{2}$ output and $\mathrm{O}_{2}$ uptake. Usually, it is considered during physical stress, providing information about AT and predominant fuel utilization (with the acknowledged limitation of not considering protein utilization). The classical criteria for detecting AT are the following:1) analysis of the straight-line relations of $\mathrm{VCO}_{2} \mathrm{vs} \mathrm{VO}_{2}$ (V-slope method) [23]; 2) inflection point on the minute ventilation $\left(\mathrm{V}_{\mathrm{E}}\right)$ vs oxygen consumption $\left(\mathrm{VO}_{2}\right)$ diagram; 3 ) point of increase in end-tidal $\mathrm{VO}_{2}\left(\mathrm{PETO}_{2}\right)$; 4) point of increase in the ventilatory equivalent of $\mathrm{O}_{2}\left(\mathrm{~V}_{\mathrm{E}} / \mathrm{VO}_{2}\right)$ without a concomitant increase in the ventilatory equivalent of $\mathrm{CO}_{2}\left(\mathrm{~V}_{\mathrm{E}} / \mathrm{VCO}_{2}\right)$ [23].

Human muscles contain both slow twitch fibers (type 1) more fat burning, and fast twitch fibers (type 2) with a preference for carbohydrate. Lower exercise intensity recruits more type 1 fibers while highest intensities recruit an higher percentage of type 2 fibers, which are added to those of type $1[20]$.

This review stems from exercise testing in subjects with obesity and emphasizes the relevance of AT as a drift point in the behavior of specific hormones and mediators. Building on that physiological evidence, it will summarize the research conducted up to date on the effects of physical training on metabolism and body composition of individuals with obesity at work outputs below and beyond AT. Finally, we will propose a combination of Aerobic Training along with Aerobic plus Anaerobic Training as appropriate exercise prescription in subjects with obesity undergoing rehabilitation.

The rationale of combining Aerobic and Anaerobic training (which entails "playing around the anaerobic threshold") is particularly relevant in the COVID prevention and in the post-COVID rehabilitation of subjects with obesity. This is because training our muscles to dispose more quickly of lactic acid will increase the aerobic power of the mitochondria, and specific training programs tailored for individuals with obesity may improve their immunomodulatory capacity
[7]. Moreover, sedentariness caused by lockdown periods has a deconditioning effect also in unaffected individuals, although it determines a striking burden in individuals with obesity or diabetes [24]. Recently, high-intensity interval training (HIIT) (brief bursts of vigorous intensity interspersed with periods of rest or low-intensity exercise) has been tested in subjects with obesity, showing positive effects on aerobic fitness and cardiovascular protection [25].

\section{Literature search}

We searched the PubMed database for relevant literature. The search on obesity and AT was performed focusing in parallel on: 1) behavior of hormones and mediators during exercise testing; 2) effects of training at different intensities (that is below AT vs below and beyond AT); 3 ) effects of bouts of high-intensity exercise in relation to COVID-19.

We used the following keywords for the first search topic: (obesity AND adults AND exercise AND anaerobic threshold) AND plasma catecholamine OR epinephrine OR norepinephrine OR lactic acid OR potassium OR growth hormone OR GH OR leptin. Subsequently, a second search topic with the following keywords was performed: (obesity AND adults AND exercise AND anaerobic threshold AND physical training) AND growth hormone OR GH OR nonesterified fatty acids OR NEFA OR insulin resistance OR lactic acid OR leptin OR body composition. Finally, as last topic, a search using as keywords (Obesity AND COVID-19 AND High Intensity Exercise) was performed.

Only peer-reviewed articles written in English were included in the list of retrieved scientific studies. The final screening was based on the relevance of the identified items. Twelve articles were considered in the first search topic (McMurray RG et al. 2005, Connolly DAJ et al. 2012, Wasserman K 1984, Salvadori A et al. 1992, Salvadori A et al. 1991, Cahill BR et al. 1997, Salvadori A et al. 2003, Salvadori A et al. 2008, Hamilton MT et al. 2000, Salvadori et al. 2004, Felsing NE et al. 1992, Koppo K et al. 2010), ten articles were considered in the second search topic (Tamai M et al. 1993, Weltman A et al. 2008, Sideman L et al. 2002, Salvadori A et al. 2010, De Glisezinski I et al. 2003, Dengel DR et al. 1996, Salvadori et al. 2014, Salvadori A et al. 2015, Jelleyman C et al. 2015, Lanzi S et al. 2015) and five articles were considered in the third search topic (RahmatiAhmadabad S et al., 2020, Yang S, 2020, Wang M, 2020, Baena Morales S 2021, Kemmler W, 2021).

Considering these retrieved papers and the experience of our multidisciplinary team, we report on the following observations linking physical activity to anaerobic threshold in subjects with obesity, before and during COVID-19 pandemic, speculating on a potential protection from COVID-19 of above AT or of mixed aerobic and anaerobic training. 


\section{Anaerobic threshold in subjects with obesity and normal subjects}

During a progressive exercise testing, AT (former threshold) represents a "drift point" for many indexes (catecholamine, lactic acid, potassium and growth hormone).

\section{Plasma catecholamine pattern during exercise in obesity: its potential ominous role in COVID-19}

Physical stress causes activation of the SNS. This elicits an increase in plasma catecholamine, which in turn contributes to increasing the heart rate, arterial pressure, and myocardial contractility [26]. In addition, due to SNS innervation of adipose tissue, physical stress promotes lipolysis and release of glycerol and free fatty acids (NEFA) into the circulation [27].

Exercise intensity is a key determinant of the catecholaminergic response. Power outputs exceeding the AT are associated with an exaggerated catecholamine activation [28] with an exponential rising of epinephrine (E) and norepinephrine (NE) concentrations [29]. Factors like degree of fitness, muscle conditioning, and degree of involvement of the muscular mass can influence catecholamine response [26-29].

In contrast to the lean subjects, individuals with obesity show a trend toward higher catecholamine response for lower work outputs before AT, while a lower catecholamine response for higher work outputs beyond AT [30].

During a progressive cycle-ergometer test in untrained young normal subjects, plasma epinephrine levels have been shown to increase by $180 \%$ from rest to AT, and by $950 \%$ from rest to maximal sustainable peak activity. In contrast, in subjects with obesity, the increments in epinephrine levels were 205\% from rest to AT and 335\% from rest to maximal sustainable peak activity.

Norepinephrine concentration increased, respectively, $215 \%$ and $550 \%$ in subjects with obesity while $162 \%$ and $920 \%$ in lean age-matched controls [30]. These data partly confirm those of Gustafson et al. [31] and may suggest that obesity can elicit different catecholamine responses depending on the intensity of the physical stress.

The altered catecholamine kinetics present in individuals with obesity at low workloads (with a more pronounced increment of both epinephrine and norepinephrine) may predispose to cardiac complications during COVID-19. In fact, firstly an altered catecholamine physiology was demonstrated during SARS-CoV2 infection [32]. Secondly, the high prevalence of Takotsubo syndrome in COVID19 patients was documented. Takotsubo syndrome, which is often fatal, is characterized by ECG alterations (ST elevations, precordial $\mathrm{T}$ wave inversion), normal or nearnormal troponin and high level of catecholamine. This syndrome may lead to acute cardiac failure with low cardiac output [33]. Thirdly, the psychological distress undermining our lives in COVID-19 era may increase the incidence of Takotsubo syndrome, mainly in individuals with obesity with a higher catecholamine response than lean subjects at low workloads [34]. In other words, individuals with obesity usually show higher catecholamine levels that lean counterparts during everyday life activities and this pattern may expose them to higher risk of cardiovascular complications during COVID, such as Takotsubo syndrome.

\section{Plasma lactic acid and plasma potassium during exercise in subjects with obesity: the potential role on prognosis of COVID-19}

As stated above, plasma lactic acid increases when metabolism has shifted toward nonoxidative pathway, beyond the "lactate threshold," to increase ATP production.

Lactic acid increases during a progressive exercise. In subjects with obesity, exercise beyond AT increases lactic acid production less than in controls at similar maximal power outputs [35]. This blunted response is due to the wellknown insulin resistance, typical of subjects with obesity. Noticeably, increases in lactate anions play an important role in changing hydrogen ion concentrations in plasma and muscles [36] and, in turn, this could be relevant for the control of ventilatory function like ventilation during exercise. In the basal state, individuals with obesity produce more lactic acid than lean subjects do, mainly from chronically hypoxic adipocytes [37]. The high lactic acid outflow contributes to the development of blood acidosis and constitutes a predisposing factor to multi-organ failure in patients with obesity affected by COVID-19 [8].

Physical stress elicits a release of potassium ions $\left(\mathrm{K}^{+}\right)$ from contracting muscles, which is proportional to the entity of work [38]. Potassium release depends on a mismatch between the potential charge of sarcolemma during muscle contraction and the ability of the $\mathrm{Na}^{+}-\mathrm{K}^{+}$pump to keep pace with the rate of $\mathrm{K}^{+}$loss and/or to a simultaneous acid-base change due to reduction in nondiffusible intracellular anions linked to phosphocreatine hydrolysis [39]. $\mathrm{K}^{+}$reuptake by the $\mathrm{Na}^{+}-\mathrm{K}^{+}$pump in contracting and noncontracting muscles, as well as kidneys, is able to counterbalance $\mathrm{K}^{+}$release [40]. It is associated with the activity of $\alpha$ and $\beta$-adrenergic receptors, which, respectively, reduce and increase the $\mathrm{Na}^{+}-$ $\mathrm{K}^{+}$pump activity [40]. Through $\beta$-adrenergic stimulation, this catecholamine-driven $\mathrm{K}^{+}$ions reuptake promotes and protects against hyperkalemia [40].

Static muscular contractions increase heart rate and arterial pressure [41] by means of a reflex initiated by the 
stimulation of Group III and IV afferents whose terminals are located in the interstitium of the working muscle [42]. The accumulation of $\mathrm{K}^{+}$in the interstitium of the muscle is considered a possible important mechanism by which contractions activate Group III and IV afferents [43]. Both in normal weight subjects and individuals with obesity, a progressive physical exercise causes significant increases in plasma $\mathrm{K}^{+}$, although increases are lower in subjects with obesity [30] (Fig. 1).

Structural modifications in peripheral organs may contribute to the regulation of cardiovascular and respiratory parameters variables $[44,45]$. In fact, quadriceps muscular biopsies in subjects with obesity are characterized by fiber hypertrophy and intracellular accumulation of neutral lipids [46-48]. Fiber hypertrophy has been associated with a higher density of the $\mathrm{Na}^{+}-\mathrm{K}^{+}$pump sites [49]. This finding possibly explains the lower increase in plasma $\mathrm{K}^{+}$in subjects with obesity seen during progressive exercise. Moreover, subjects with obesity show lower increases in plasma $\mathrm{K}^{+}$ at work outputs below AT with respect to controls, while higher increases than controls are seen in individuals with obesity beyond AT [30] (Fig. 1). This apparently divergent behavior could be explained by the greater muscular mass of subjects with obesity involved in the physical exercise. Higher muscle mass intensifies $\mathrm{E}$ and NE release at the lower outputs, and a lower increase in plasma $\mathrm{K}^{+}$observed before AT could be due to enhanced muscular $\mathrm{K}^{+}$reuptake by $\beta$ receptors. Beyond AT, the lower increase in plasma $\mathrm{E}$ and $\mathrm{NE}$ is coupled with a greater increase in plasma $\mathrm{K}^{+}$. The opposite seems to occur in controls [30].

The altered $\mathrm{K}^{+}$kinetics of individuals with obesity may concur in worsening the hypopotassemia present in COVID19 [50]. In fact, the occupancy of ACE2 receptors by SARS$\mathrm{CoV} 2$ binding via the viral $S$ protein causes an imbalance in the Renin-Angiotensin-System determining a prevalence of ACE1 (which increases RAS activity) [50]. The increase in
RAS activity leads to an increase in aldosterone and consequent increase in renal excretion of $\mathrm{Na}^{+}$, water and $\mathrm{K}^{+}$, ending up in hypopotassemia, which may last much longer after remission of COVID-19 [51]. An imbalance of the RAS system may also cause a pro-thrombotic state contributing to the multi-organ failure based on metabolic acidosis [52].

Plasma $\mathrm{K}^{+}$is also involved in the incremental ventilatory response during physical activity [53, 54]. In subjects with obesity undergoing a progressive incremental exercise, the ventilatory response is less robust than in controls. The difference is maximal when ventilation has adjusted for the fat-free mass [35].

To summarize the role of lactate and $\mathrm{K}^{+}$, they are involved in the regulation of $\mathrm{V}_{\mathrm{E}}$ with a peculiar pattern linked to the morphology of muscle fibers (hypertrophy) and insulin resistance (basal increase in lactate and reduced increase in lactic acid at maximal work outputs) $[35,53$, 54]. The alterations of both $\mathrm{K}^{+}$and potassium kinetics may play specific roles to worsen the prognosis of COVID-19 in subjects with obesity.

\section{Growth hormone (GH) during exercise in subjects with obesity: its potential role in COVID-19 prognosis}

$\mathrm{GH}$ is one of the key regulators of body fat and lean tissue. A variety of conditions affect GH secretion like, gender, pubertal stage, age, sleep, nutritional status, body composition, body temperature, fitness, gonadal steroids, insulin and IGF-1 [55-57].

Physical activity stimulates GH secretion [58]. The magnitude of GH response to exercise depends on its duration and intensity, as well gender, fitness and age [59]. An open question regards the hypothesis that GH response implies a threshold of exercise intensity, or a linearly increase along with increasing intensity of work [60-62].
Fig. 1 COVID-19 shifts metabolism toward anaerobic glycolysis eventually inducing metabolic acidosis and hypopotassemia. Bouts of physical exercise above AT cause a positive modulation of hormones and metabolites like, growth hormone, lactic acid, $\mathrm{H}^{+}, \mathrm{K}^{+}$

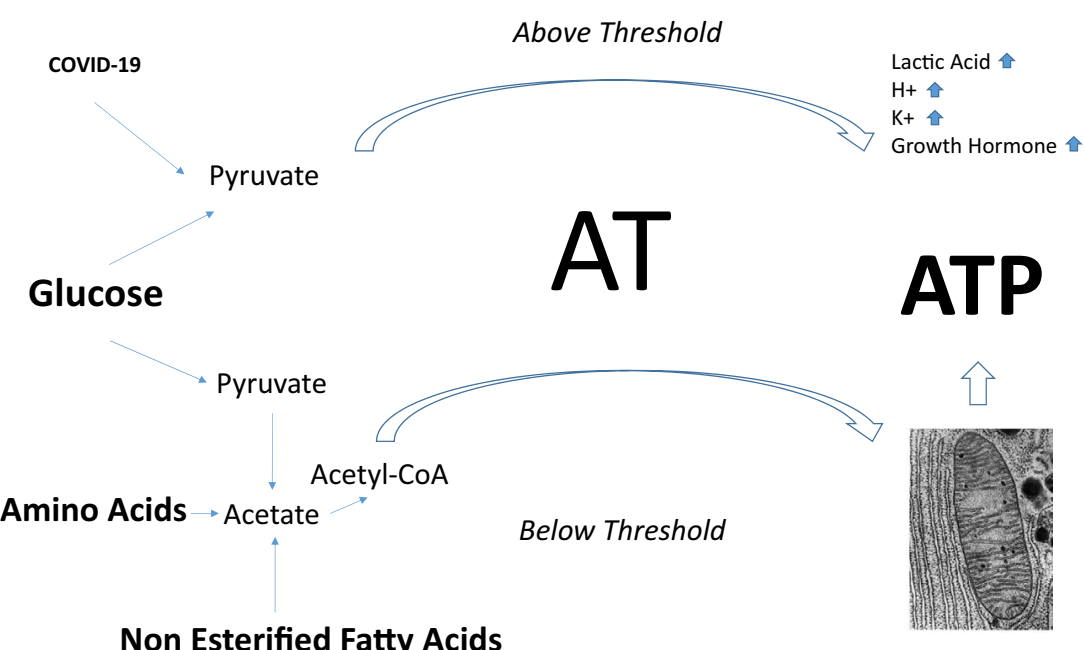

Springer 
Obesity blunts the GH response to exercise [55]. Similarly, abdominal visceral fat has been identified as a responsible for the reduction both in spontaneous and stimulated GH secretion $[55,56]$. In lean subjects, during a progressive exercise, a significant GH increase is detectable from rest to AT, and a further significant increase vs rest and vs AT up to maximal activity. In subjects with obesity no significant GH increase occurs from rest to AT, while a slight but significant GH increase is seen from AT to peak activity when expressed as area under the curve (AUCs). Compared to lean controls, GH response to exercise is null before AT, and significantly lower beyond AT in subjects with obesity [63].

The blunted GH response may partially explain the greater severity of COVID-19 illness of individuals with obesity, mainly if they are elderly and of male sex [64]. The proposed underlying mechanism is a defective immunomodulatory effect in GH deficient individuals [64]. In fact, growth hormone plays a fundamental role in immune modulation. Therefore, exercising above the AT may increase GH response in subjects with obesity, and it is conceivable that the higher GH response may induce a positive immunomodulatory effect [64].

\section{Leptin during exercise in subjects with obesity: relationship with COVID-19}

Leptin is a hormone involved in the regulation of body weight and satiety [65], mainly secreted by adipocytes [66, 67]. It decreases when energy intake is restricted, and increases as body fat accumulates [67]. It is well known that obesity is associated with an increase in circulatory leptin levels and prompts a condition of leptin resistance [67]. Both acute physical exercise and prolonged physical training promote a decrease in plasma leptin [68-70]. Growth hormone is a factor able to contrast the extent of leptin reduction due to physical stress [71], as well as the increase in intracellular products from glucose metabolism from bouts of work beyond AT, in absence of improvement in insulin sensitivity for glucose [72].

The higher leptin levels present in individuals with obesity may play a role in the severity of COVID-19 [73]. In fact, besides regulating appetite and metabolism, leptin also signals via the Jak/STAT and Akt pathways and modulates T cell function. Recent studies demonstrated that leptin upregulates expression of inflammatory cytokines in monocytes, and potentially contributes to ensuing the so-called cytokine storm, which often preludes to a negative outcome of COVID19 [74].

\section{Fat $_{\text {max }}$ and high-intensity interval training (HIIT) in subjects with obesity: its potential role in COVID-19 prevention}

Herein, we have considered AT as a corner stone because it implicates modifications in hormones and mediators responses during a progressive exercise. Some authors have focused on exercise modalities utilizing other training models, like continuous work at Fat ${ }_{\text {max }}$ (exercise training at intensity eliciting maximal fat oxidation) and highintensity interval training (HIIT).

Fat $_{\max }$ exercise training is performed at $60-70 \%$ of maximal heart rate. This, particularly in subjects with obesity, could be an exercise threshold already across, if not clearly beyond, the achievement of AT [75].

About HIIT, repeated bouts of maximal exercise have shown a decreasing activity of glycolysis, with a shift toward oxidative phosphorylation [76]. Thus, HIIT is already considered as a modality of aerobic training [76].

In subjects with obesity, adaptation to moderate and high-intensity interval exercise can differ interindividually and high-intensity exercise training sessions are not feasible for all patients with obesity undergoing a training program, especially in subjects with class III obesity [77].

We previously showed that in subjects with second and third class of obesity, eight cycling sessions (spread over 2 weeks) of a moderate-intensity continuous training were both effective in improving aerobic fitness and fat oxidation rates during exercise [78]. In this study, HIIT had tendency toward promoting a more marked increase in $\mathrm{VO}_{2 \max }$ compared to $\mathrm{Fat}_{\max }$ training $(+8 \%$ and $+4 \%$, respectively). This improvement is likely related to exercise intensity [79] and highlights the following: 1) HIIT is a feasible and time-efficient training in class II and III subjects with obesity, as previously shown in overweight and class I subjects with obesity [80]; 2) in those obesity categories, HIIT improves aerobic fitness [81-83]; 3) promoting HIIT early after initiation of training programs can help to reverse the low aerobic fitness in individuals with obesity [84]. While HIIT seems preferable at Fat ${ }_{\text {max }}$ when compared to moderate-intensity training, only the latter induced a significant reduction in fasting insulin and insulin resistance [85], suggesting the importance of exercise duration for improving insulin sensitivity in subjects with obesity. The insulin-sensitizing effect of Fat ${ }_{\max }$ could be related to decreased levels of plasma non-esterified acids (NEFA) [86, 87]. Finally, considering the need of increasing training variety and adherence in the real world setting [88], HIIT and moderate-intensity continuous training may be two complementary training tools.

In relation to COVID-19, both Fat max $_{\text {ax }}$ and HIIT seem appropriate trainings modalities in subjects with obesity. 
The former, with a work intensity at $60-70 \%$ of maximal heart rate reach the AT in individuals with obesity, and all the benefits illustrated in the previous chapter. The latter (HIIT) being a modality of aerobic training reduces the leptin level in subjects with obesity, ameliorating the negative effect of leptin on immune modulation [73, 74]. In particular, in a social condition of lockdown and distancing with gyms and sites for aggregation unavailable, HIIT, namely a training model requiring less time to obtain the metabolic and immunomodulatory effects, seems the most appropriate way of exercising.

\section{Playing around the anaerobic threshold: a tool against Sars-cov2 infection and complications}

Regular physical exercise associated with diet is fundamental strategy in the management and rehabilitation of subjects with obesity. The American College of Sport Medicine recommends the addition of resistance exercise to a regular program of aerobic training [89]. Mild, aerobic exercise increases lipolysis by means of increased plasma catecholamine and lowers plasma concentrations of insulin an antilipolytic hormone, while plasma NEFA concentrations drop after exercise [90-93].

To our knowledge, only few studies analyzed the effects of low- or high-intensity training in individuals with obesity. To investigate the effects of moderate-intensity training below and beyond AT on body weight, body composition and metabolism in adult subjects with obesity, this protocol has been used to better clarify the isolate impact of physical activity on weight loss and biochemical modifications at different work outputs. It could be also adopted for the rehabilitation of post-COVID-19 patients with obesity, based on all considerations previously discussed.

Two experimental training conditions have been tested: (a) moderate-intensity training below AT and (b) moderateintensity training with a single bout of high-intensity exercise, beyond AT. The study protocol was conducted during a 4-week hospital stage (two cycle-ergometer sessions/day of $1 / 2 \mathrm{~h}$, for 6 days/week) with one group training at constant work-output attaining $70 \%$ AT heart rate, while the other underwent 25 min training at $70 \%$ AT and a final exercise bout of $5 \mathrm{~min}$ at $85 \%$ of maximal heart rate. Noticeably, there was no significant difference in the total amount of performed work when referred to fat-free mass between group (a) and group (b) after the period of training [94].

After the 4-wk reconditioning program, both types of exercise determined an increase in AT, of the maximal peak of activity, of the $\mathrm{VO}_{2}$ max, associated with a decrease in body weight. In particular, the decrease in fat mass was significantly higher following moderate-intensity training plus single bout of high-intensity exercise (b) compared to exercise performed only at moderate intensity (a) [93].

\subsection{Growth hormone (GH) after training in individuals with obesity}

As reported before, subjects with obesity achieve a null GH response exercising below AT, and a clearly lower response when they exercise beyond AT when compared to lean subjects. This confirms that AT appears as a point-break for the modification in the $\mathrm{GH}$ response to exercise in subjects with obesity [63].

After four weeks of training, the GH response to exercise of moderate-intensity training plus single bout of highintensity exercise (b) was higher than $\mathrm{GH}$ response following only moderate intensity (a) in individuals with obesity. In fact, adding single bouts of strenuous work at the end of each regular session of moderate intensity is able to evoke a significantly higher GH secretion in response to exercise intensity exceeding AT as shown in Fig. 2 [63]. The restoration of GH secretion will improve the immunomodulatory capacity in individuals with obesity.

\section{Non-esterified fatty acids (NEFA), insulin resistance, lactic acid after training in subjects with obesity.}

Aerobic training reduces circulating NEFA [90-93] both in resting condition and during physical activity (Fig. 3). An increasing amount of lipids oxidized during exercise may cause a negative balance between a slow mobilization of fatty acids from adipose tissue and their rapidly increased extraction by the skeletal muscle in activity [95]. Moreover, aerobic training reduces insulin resistance (HOMA 2-B) and increases lactic acid during exercise beyond AT [93]. In accordance with other studies [96, 97], these results suggest

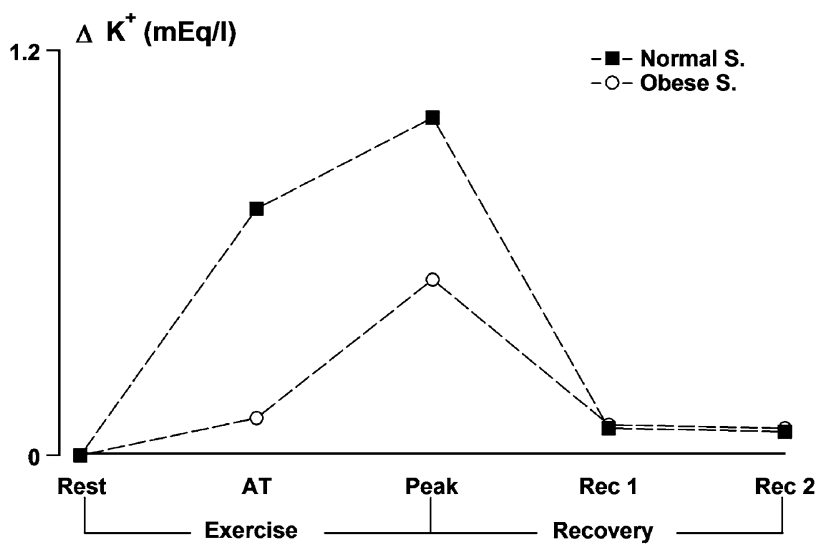

Fig. $2 \mathrm{~K}^{+}$modification during exercise testing before and after anaerobic threshold 

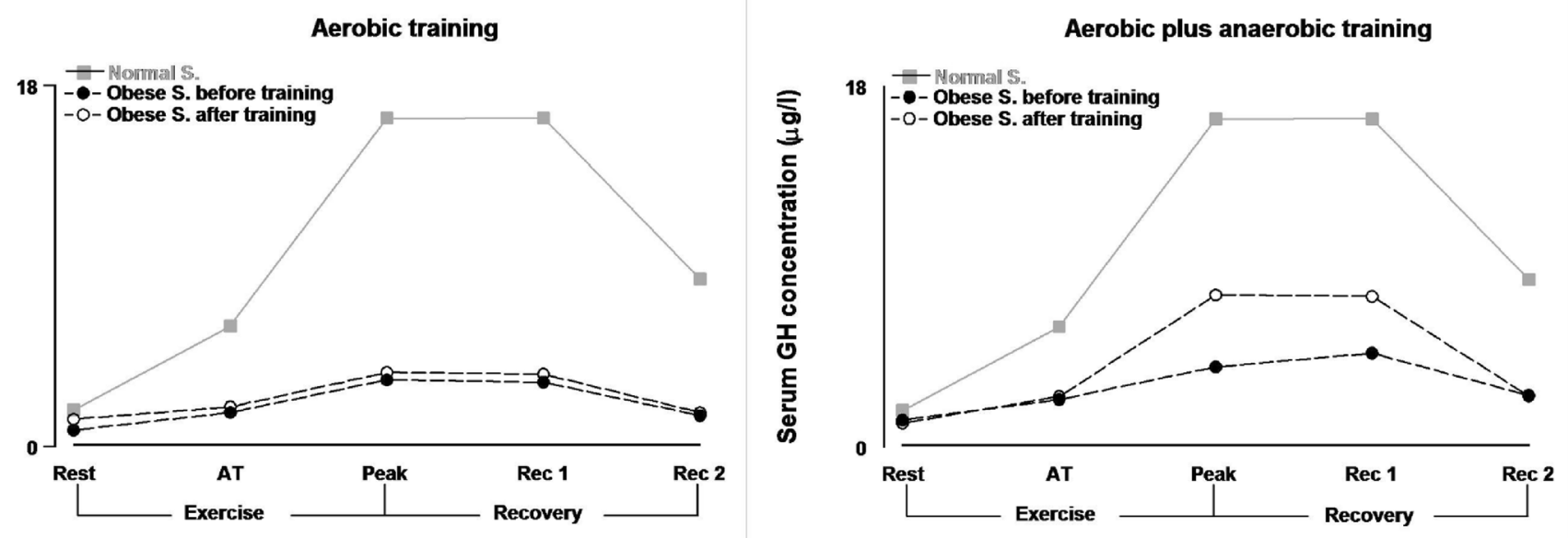

Fig. 3 Serum GH concentration during exercise testing before and after aerobic training (left panel), before and after aerobic plus anaerobic training (right panel) in subjects with obesity vs normal subjects

that both mitochondrial oxidative capacity and glucose utilization have improved following moderate aerobic exercise.

In contrast to moderate-intensity training (a), a period of moderate training with brief periods of work beyond AT (b) seems to promote different modifications, like an increase in circulating NEFA (Fig. 3), a significantly higher fat mass loss, no change in HOMA 2-B, a total lower level in lactic acid during exercise [93]. Lactate is a major energy source, and its lower levels during exercise after aerobic and anaerobic training may even be due to a higher clearance, as observed in trained athletes [98]. Training the subjects with obesity to better dispose lactic acid and potassium is a protective mechanism in case of SARS-CoV2 infection $[7,8,30]$.

Overall, a moderate-intensity training constantly below AT (a) seems to promote an improvement in NEFA utilization and, at the same time, an improvement in insulin sensitivity, with a poor fat mass reduction [93]. A moderateintensity training with bouts of high intensity (b) (that is beyond AT) seems to yield no clear improvement in metabolic profile, while fat mass reduction becomes more efficient [93]. It is conceivable that the physical stress due to physical exercise beyond AT does not improve insulin sensitivity, while the increased spillover of lipolytic mediators like catecholamines and $\mathrm{GH}$ promotes a lipid mobilization which exceeds their dynamic utilization [93].

\subsection{Leptin after training in subjects with obesity}

After a period of training, serum leptin decreases in obesity together with fat mass. Aerobic exercise (a) promotes a significant decrease in leptin and a slight fat mass loss, while aerobic and anaerobic activity and (b) promotes a slight reduction in leptin with a higher fat mass loss [94].
In the absence of dietary restrictions, physical training decreases leptin hypersecretion and fat mass. Leptin decrease seems to be related only to the intensity of performed work, but dissociated from fat mass loss after training, although the relationship between physical exercise, leptin and fat mass is very complex [99, 100].

The robust reduction in leptin after moderate exercise training alone (a) linearly correlates with the total amount of performed work. The higher the amount of work, the higher the reduction in leptin [94].

The slight reduction of leptin after moderate plus highintensity training (b) implies an opposite trend: the higher the amount of work, the lower the leptin reduction [94].

This consideration may agree with the notion that factors like $\mathrm{GH}$ as well as an increase in intracellular products of glucose metabolism (e.g., high lactic acid due to work beyond AT) contrast the reduction in leptin caused by physical stress.

\section{Can physical exercise around the anaerobic threshold prevent COVID-19 infection and serious?}

Since AT is a point-break leading to the release of metabolites and hormones affecting the immunomodulatory capacity of individuals with obesity, it is mandatory to exceed the AT level performing specific training programs. We speculate that adding short bouts of exercise above AT to medium-intensity continuous exercise below AT (50\% of $\mathrm{VO}_{2}$ max) may permanently reduce lactic acid and $\mathrm{H}^{+}$[93] and increase growth hormone secretion (Fig. 4) [63]. Those hormone and metabolite changes will improve the host defenses against viral infections and reduce the likelihood 

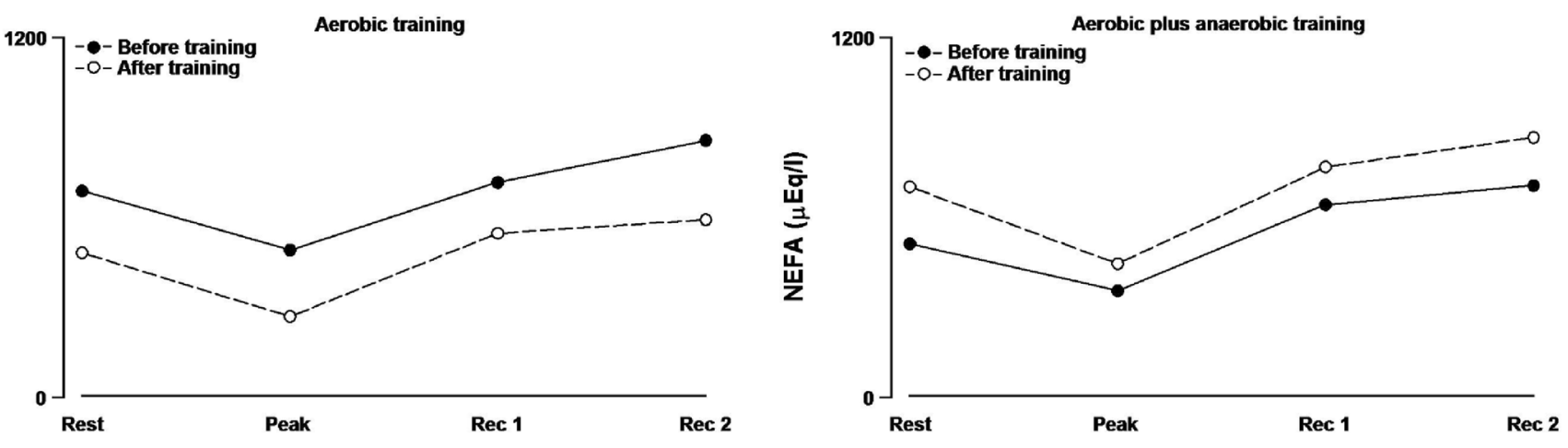

Fig. 4 Plasma NEFA concentration during exercise testing before and after aerobic training (left panel), before and after aerobic plus anaerobic training (right panel) in subjects with obesity

of metabolic acidosis following SARS-CoV-2 contagion. In contrast, the impact of a lower reduction of leptin levels on the immunomodulatory capacity of individuals with obesity, obtained adding bouts of exercise above the AT, is hard to explain at present.

\section{Physical exercise and recovery from cardiopulmonary sequelae of COVID -19 in patients with obesity}

Based on the above considerations, the opportunity of training programs including bouts of exercises above the AT applies also after a COVID-19 illness in individuals with obesity. In fact, firstly, cardiopulmonary sequelae are frequent in the post-COVID period, and frail individuals, like subjects with obesity, necessitate of muscular and cardiopulmonary rehabilitation. Secondly, a prolongation of symptoms like asthenia, muscle aches, intermittent lowgrade fever, shortness of breath and cognitive dysfunction may persist up to 4-6 months after the negativization of SARS-CoV2 throat swab, identifying the newly called "Long-COVID" syndrome [101]. Thirdly, the possibility of re-infection with a new viral variant [102]. All those factors make useful the implementation of physical exercise programs both to prevent re-infection and to rehabilitate postCOVID individuals with obesity.

\section{Conclusions}

In all vertebrates, the start of any movement is an anaerobic process, being the anaerobic energy system the source of emergency-response energy. During evolution of species, the anaerobic power and capacity of vertebrate's genera has been an essential component of survival [103] pinpointing on the importance of the anaerobic threshold regulation.
However, current understanding of role of AT is generally limited as the knowledge of the underlying complex interplay of biochemical reactions and responses [104]. Nevertheless, in physical rehabilitation AT can be easily monitored via heart rate and its changes during training and testing and provides relevant information on relative and absolute intensity of work, including efficiency and safety [104]. Herein, we showed that AT can be considered not only a switch for respiratory gases and for lactic acid, but, presumably, it also represents a trigger for the activity of various hormones and biochemical mediators. The occurrence of COVID-19 pandemic prompted the present work, and the suggestion of adding bouts of exercise above AT is strictly enforced in subjects with obesity both to prevent SARS-CoV2 infection and to combat COVID-19 sequelae and re-infection during rehabilitation periods.

In accordance with recent reports, scientific evidence emphasizes the effectiveness of physical training in the treatment of obesity, particularly during global pandemics [105]. This review reports on the differential response of lean subjects and individuals with obesity during different modalities and intensities of physical exercise as the pattern and modifications of mediators, hormones and metabolites are concerned. We aimed to describe the different biologic responses to physical stress when exercising "up and down" the anaerobic threshold and to suggest a prescription of physical activity tailored for rehabilitation of individuals with obesity.

The distillation of our review in a practical suggestion entails the opportunity to prescribe a period of moderate (below AT) plus high-intensity (beyond AT) training as first approach in subjects with obesity without major comorbidities. The rationale behind this approach is to search for a maximal stimulation of lipolysis. Subsequently, it is conceivable to stimulate metabolic rate with a prolonged moderate-intensity training alone, to further improve fat loss and ameliorate insulin and leptin resistance. This approach would be particularly valuable for individuals with obesity 
during the COVID-19 pandemic as concerns both prevention and follow-up. Interestingly, the considerations concerning COVID-19 might be extended to other viral pandemics, e.g., the influenza virus's pandemics [8]. This is because adding bouts of high-intensity exercise (above AT) will induce positive immunomodulatory changes.

Several recent reports are along the line of the proposed training program for the prevention of COVID-19 [106]. In particular, older individuals may benefit of appropriate HIIT programs during COVID-19 pandemic [107]. In a multinational study, Wang et al. propose HIIT along with moderate aerobic training $\left(\mathrm{Fat}_{\max }\right)$ as an approach that reduces infection rates, underlying pathologies and chance of mortality associated with COVID-19 [108], while Baena-Morales identifies several training modalities for different target groups [109]. Finally, Yang et al. clearly showed the dramatic impact on body weight of forced sedentariness during lockdown periods [110].

Clearly, there are also some limitations and contraindications to the suggested training program: firstly, individuals with obesity may have several comorbidities like hypertension or ischemic heart disease; secondly, subjects with grade II and III obesity may have biomechanical impediments to perform high-intensity physical activity; thirdly, the psychological reluctance of subjects with obesity to perform physical activity.

In conclusion, moderate- and high-intensity exercise training may be two complementary tools, to optimize the management of individuals with obesity, in the outpatient or in the rehabilitation ward settings, particularly useful during COVID-19 pandemic.

Acknowledgements We gratefully remember Prof. Erminio Longhini for his fundamental presence and lasting collaboration which has stimulated and extended our medical research and careful attention to the patient

\begin{abstract}
Authors' contributions AS designed the paper, and contributed to Introduction and Discussion; PF contributed to design of the paper, Literature search and Plasma catecholamine measurement; FC contributed to Literature search, Plasma lactic acid and Plasma potassium measurements; MC contributed to Plasma lactic acid and Plasma potassium measurements; IT and PP contributed to Growth hormone and leptin measurement; LL, IT and AF: contributed to design and final content and grammatical revision of the paper, plasma catecholamines and NEFA measurement; AB contributed to NEFA and insulin resistance assessment; SL contribution to Obesity protocol design and training related to $\mathrm{Fat}_{\max }$ and High-Intensity Interval (HIIT). All authors have approved the final article.
\end{abstract}

Funding Open access funding provided by Università degli Studi di Milano within the CRUI-CARE Agreement. This work was partially supported by the Italian Ministry of Health, Ricerca Corrente, IRCCS MultiMedica and IRCCS Istituto Auxologico Italiano.

\section{Declarations}

Conflict of interest The authors have no conflicts of interest to declare.

Ethical approval The authors have no ethical conflicts to disclose.

Informed consent All human studies quoted in the present review were approved by Ethics Committee of Istituto Auxologico Italiano, Milan, Italy and all subjects signed an informed consent.

Open Access This article is licensed under a Creative Commons Attribution 4.0 International License, which permits use, sharing, adaptation, distribution and reproduction in any medium or format, as long as you give appropriate credit to the original author(s) and the source, provide a link to the Creative Commons licence, and indicate if changes were made. The images or other third party material in this article are included in the article's Creative Commons licence, unless indicated otherwise in a credit line to the material. If material is not included in the article's Creative Commons licence and your intended use is not permitted by statutory regulation or exceeds the permitted use, you will need to obtain permission directly from the copyright holder. To view a copy of this licence, visit http://creativecommons.org/licenses/by/4.0/.

\section{References}

1. Troisi RJ, Weiss ST, Parker DR, Sparrow D, Young JB, Landsberg L (1991) Relation of obesity and diet to sympathetic nervous system activity. Hypertension 17:169-177

2. Jones PP, Davy KP, Alexander S, Seals DR (1997) Age-related increase in muscle sympathetic nerve activity is associated with abdominal adiposity. Am J Physiol 272:E976-E980

3. Seals DR, Bell C (2004) Chronic sympathetic activation: consequence and cause of age-associated obesity. Diabetes 53:276-285

4. Arner P (1999) Catecholamine-induced lipolysis in obesity. Int J Obes Relat Metal Disord 23(Suppl. 1):S10-S13

5. McMurray RG, Hackney AC (2005) Interactions of metabolic hormones, adipose tissue and exercise. Sports Med 35(5):393-412

6. McMurray RG, Hackney AC (2002) Endocrine responses to exercise and training. In: Garrett WE, Kirkendall DT (eds) Exercise and sport science. Lippincott Williams \& Wilkins, Philadelphia (PA), pp 135-162

7. Codella R, Chirico A, Lucidi F, Ferrulli A, La Torre A, Luzi L (2020)The immune-modulatory effects of exercise should be favorably harnessed against COVID-19. J Endocrinol Invest 3:1-4. Doi: https://doi.org/10.1007/s40618-020-01403-5. Epub ahead of print. PMID: 32885340; PMCID: PMC7471548.

8. Luzi L, Radaelli MG (2020) Influenza and obesity: its odd relationship and the lessons for COVID-19 pandemic. Acta Diabetol 57(6):759-764. Doi: https://doi.org/10.1007/s00592-020-015228. Epub Apr 5. PMID: 32249357; PMCID: PMC7130453.

9. Lauer MS, Anderson KM, Kannel WB, Levy D (1991) The impact of obesity on left ventricular mass and geometry. JAMA 266:231-236

10. Naimark A, Cherniack RM (1960) Compliance of the respiratory system and its components in health and obesity. J Appl Physiol 15:377-382

11. Salvadori A, Fanari P, Mazza P, Agosti R, Longhini E (1992) Work capacity and cardiopulmonary adaptation of the obese subject during exercise testing. Chest 101:674-679

12. Wasserman K (1984) The anaerobic threshold measurement to evaluate exercise performance. Am Rev Respir Dis 129(Suppl. 2):S35-S40 
13. Brunani A, Lanzi S, Codecasa F et al (2017) Vascular dynamics and peripheral oxygen uptake in obese individuals during progressive physical exercise. Respiration 94:493-500

14. Salvadori A, Fanari P, Cavestri R, Mazza P, Baudo S, Longhini E (1991) Relationship between body mass and tolerance to physical stress in obese patients. Respiration 58:311-315

15. Jeukendrup AE, Saris WHM, Wagenmakers AJM (1998) Fat metabolism during exercise: a review-part II: regulation of metabolism and the effects of training. Int J Sports Med 19:293-302

16. Wasserman K, Whipp BJ, Koyal S, Beaver WL (1973) Anaerobic threshold and respiratory gas exchange during exercise. J Appl Physiol 35:236-243

17. Tamai M, Kubota M, Ikeda NK et al (1993) Usefulness of anaerobic threshold for evaluating daily life activity and prescribing exercise to the healthy subjects and patients. J Med Syst 17:219-225

18. Vallier JM, Bigard AX, Carrè F, Eclache JP, Mercier J (2000) Determination des seuils lactiques et ventilatoires. Position de la Societè Francaise de Medicine du Sport. Sci Sports 15:133-140

19. Thomas SG, Woodhouse LJ, Pagura SM, Ezzat S (2002) Ventilation threshold as a measure of impaired physical performance in adults with growth hormone excess. Clin Endocrinol 53:351-358

20. Cahill BR, Misner JE, Boileau RA (1997) The clinical importance of the anaerobic energy system and its assessment in human performance. Am J Sport Med 25:863-872

21. Fitts RH (1994) Cellular mechanisms of muscle fatigue. Phys Rev 74:49-94

22. de Siqueira JVV, Almeida LG, Zica BO, Brum IB, Barceló A, de Siqueira Galil AG. (2020) Impact of obesity on hospitalizations and mortality, due to COVID-19: a systematic review. Obes Res Clin Pract 14(5):398-403. Doi: https://doi.org/10.1016/j.orcp. 2020.07.005. Epub 2020 Jul 23. PMID: 32736969; PMCID: PMC7377684

23. Beaver WL, Wasserman K, Whipp BJ (1986) A new method for detecting anaerobic threshold by gas exchange. J Appl Physiol 60:2020-2025

24. Luzi L, Carruba M, Crialesi R, et al (2021) Telemedicine and urban diabetes during COVID-19 pandemic in Milano, Italy during lock-down: epidemiological and sociodemographic picture. Acta Diabetol pp. 1-9. Doi: https://doi.org/10.1007/s00592021-01700-2. Epub ahead of print. PMID: 33740123; PMCID: PMC7977495

25. Campbell WW, Kraus WE, Powell KE, et al (2019) Physical activity guidelines advisory committee*. High-intensity interval training for cardiometabolic disease prevention. Med Sci Sports Exerc 51(6):1220-1226

26. Dodge HT, Lord JD, Sandler H (1960) Cardiovascular effects of isoproterenol in normal subjects and subjects with congestive heart failure. Am Heart J 60:94-105

27. Lewis SF, Taylor WF, Graham RM, Pettinger WA, Schutte JE, Blomqvist CG (1983) Cardiovascular responses to exercise as function of absolute and relative workload. J Appl Physiol 54:1314-1323

28. Urhausen A, Weiler B, Coen B, Kindermann W (1994) Plasma catecholamines during endurance exercise of different intensities as related to the individual anaerobic threshold. Eur J Appl Physiol 69:16-20

29. Mazzeo RS, Marshall P (1989) Influence of plasma catecholamines on the lactate threshold during graded exercise. J Appl Physiol 67:1319-1322

30. Salvadori A, Fanari P, Giacomotti E et al (2003) Kinetics of catecholamines and potassium, and heart rate during exercise testing in obese subjects. Eur J Nutr 42:181-187
31. Gustafson AB, Farrell PA, Kalkhoff RK (1990) Impaired plasma catecholamine response to submaximal treadmill exercise in obese women. Metabolism 39:410-417

32. Gubbi S, Nazari MA, Taieb D, Klubo-Gwiezdzinska J, Pacak K (2020) Catecholamine physiology and its implications in patients with COVID-19. Lancet Diabetes Endocrinol 8(12):978-986. Doi: https://doi.org/10.1016/S2213-8587(20)30342-9. Epub 2020 Oct 28. PMID: 33128872; PMCID: PMC7598304

33. Titi L, Magnanimi E, Mancone M, et al (2020) Fatal Takotsubo syndrome in critical COVID-19 related pneumonia. Cardiovasc Pathol 51:107314. Doi: https://doi.org/10.1016/j.carpath. 2020.107314. Epub ahead of print. PMID: 33259936; PMCID: PMC7699026

34. Barbieri L, Galli F, Conconi B, et al (2021) Takotsubo syndrome in COVID-19 era: is psychological distress the key? J Psychosom Res 140:110297. Doi: https://doi.org/10.1016/j.jpsychores. 2020.110297. Epub 2020 Nov 15. PMID: 33242703; PMCID: PMC7666871

35. Salvadori A, Fanari P, Tovaglieri I et al (2008) Ventilation and its control during incremental exercise in obesity. Respiration 75:26-33

36. Kowalchuk JM, Heigenhauser GJ, Lindinger MI, Sutton JR, Jones NL (1988) Factors influencing hydrogen ion concentration in muscle after intense exercise. J Appl Physiol 65:2080-2089

37. Trayhurn $P$ (2013) Hypoxia and adipose tissue function and dysfunction in obesity. Physiol Rev 93(1):1-21. https://doi.org/10. 1152/physrev.00017.2012 (PMID: 23303904)

38. Kilburn KH (1966) Muscular origin of elevated plasma potassium during exercise. J Appl Physiol 21:675-678

39. Wasserman K, Stringer WW, Casaburi R, Zhang YY (1997) Mechanism of the exercise hyperkaliemia: an alternative hypothesis. J Appl Physiol 83:431-463

40. Lindinger MI, Sjogaard G (1991) Potassium regulation during exercise and recovery. Sport Med 11:382-401

41. Rybicki KJ, Kaufman MP, Kenyon JL, Mitchell JH (1984) Arterial pressure responses to increasing interstitial potassium concentrations in hindlimb skeletal muscles of dogs. Am J Physiol 247:R717-R721

42. Rybicki KJ, Waldrop TG, Kaufman MP (1985) Increasing gracilis muscle interstitial potassium concentrations stimulate group III and IV afferents. J Appl Physiol 58:936-941

43. Hallen $\mathrm{J}(1996) \mathrm{K}^{+}$balance in humans during exercise. Acta Physiol Scand 156:279-286

44. Mitchell JH, Victor RG (1996) Neural control of the cardiovascular system: insights from muscle sympathetic nerve recordings in humans. Med Sci Sports Exerc 28:S60-S69

45. Hamilton MT, Booth FW (2000) Skeletal muscle adaptation to exercise: a century of progress. J Appl Physiol 88:327-331

46. Salvadori A, Fanari P, Dworzak F et al (2004) Respiratory and metabolic responses during exercise and skeletal muscle morphology in obesity. Sport Sci Health 1:47-54

47. Goodpaster BH, Theriault R, Watkins SC, Kelley DE (2000) Intramuscular lipid content is increased in obesity and decreased by weight loss. Metabolism 49:467-472

48. Phillips DI, Caddy S, Ilic V et al (1996) Intramuscular triglyceride and muscle insulin sensitivity: evidence for relationship in non-diabetic subjects. Metabolism 45:947-950

49. Knochel JP, Blachley JD, Johnson JH, Carter NW (1985) Muscle cell electrical hyperpolarization and reduced exercise hyperkaliemia in physically conditioned dogs. J Clin Invest 75:740-745

50. Moreno-P O, Leon-Ramirez JM, Fuertes-Kenneally L, et al E (2020) COVID19-ALC Research Group. Hypokalemia as a sensitive biomarker of disease severity and the requirement for invasive mechanical ventilation requirement in COVID-19 pneumonia: a case series of 306 Mediterranean patients. Int J Infect Dis 
100:449-454. Doi: https://doi.org/10.1016/j.ijid.2020.09.033. Epub 2020 Sep 17. PMID: 32950739; PMCID: PMC7497734

51. Alnafiey MO, Alangari AM, Alarifi AM, Abushara A (2021) Persistent Hypokalemia post SARS-coV-2 infection, is it a life-long complication? Case report. Ann Med Surg (Lond). 62:358-361. Doi: https://doi.org/10.1016/j.amsu.2021.01.049. Epub 2021 Jan 27. PMID: 33527042; PMCID: PMC7839393

52. Luzi L, Bucciarelli L, Ferrulli A, Terruzzi I, Massarini S (2021) Obesity and COVID-19: 2 The ominous duet affecting the reninangiotensin system. Minerva Endocrinol. Doi: https://doi.org/ 10.23736/S0391-1977.20.03402-1. Epub ahead of print. PMID: 33435650.

53. Paterson DJ, Dorrington KL, Bergel DH et al (1992) Effect of $\mathrm{K}^{+}$ on ventilation in the rhesus monkey. Exp Physiol 77:217-220

54. Paterson DJ, Nye PC (1991) Effect of oxygen on potassiumexcited ventilation in the decerebrate cat. Respir Physiol 84:223-230

55. Lanzi R, Luzi L, Caumo A et al (1999) Elevated insulin levels contribute to the reduced growth hormone $(\mathrm{GH})$ response to $\mathrm{GH}$-releasing hormone in obese subjects. Metabolism 48(9):1152-1156. https://doi.org/10.1016/s0026-0495(99) 90130-0 (PMID: 10484056)

56. Kanaley JA, Weatherup-Dentes MM, Jaynes EB, Hartman ML (1999) Obesity attenuates the growth hormone response to exercise. J Clin Endocrinol Metab 84:3156-3161

57. Veldhuis JD, Liem AY, South S et al (1995) Differential impact of age, sex steroid hormones, and obesity on basal versus pulsatile growth hormone secretion in men as assessed in an ultrasensitive chemiluminescence assay. J Clin Endocrinol Metab 80:3209-3222

58. Felsing NE, Brasel JA, Cooper DM (1992) Effect of low and high intensity exercise on circulating growth hormone in men. J Clin Endocrinol Metab 75:157-162

59. Weltman A, Weltman JY, Hartman ML et al (1994) Relationship between age, percentage body fat, fitness, and 24-hour growth hormone release in healthy young adults: effect of gender. J Clin Endocrinol Metab 78:543-548

60. Eliakim A, Scheet TP, Newcomb R, Mohan S, Cooper DM (2001) Fitness, training, and the growth hormone-insulin-like growth factor I axis in prepubertal girls. J Clin Endocrinol Metab 86:2797-2802

61. Weltman A, Weltman JY, Watson Winfield DD et al (2008) Effects of continuous versus intermittent exercise, obesity, and gender on growth hormone secretion. J Clin Endocrinol Metab 93:4711-4720

62. Wideman L, Weltman JY, Hartman ML, Veldhuis JD, Weltman A (2002) Growth hormone release during acute and chronic aerobic and resistance exercise. Sport Med 32:987-1004

63. Salvadori A, Fanari P, Marzullo P et al (2010) Dynamics of GH secretion during incremental exercise in obesity, before and after a short period of training at different work-loads. Clin Endocrinol 73:491-496

64. Elkarow MH, Hamdy A (2020) A Suggested Role of Human Growth Hormone in Control of the COVID-19 Pandemic. Front Endocrinol (Lausanne) 9(11):569633. https://doi.org/ 10.3389/fendo.2020.569633 (PMID:33240216;PMCID: PMC7680968)

65. Considine RV, Caro JF (1997) Leptin and the regulation of body weight. Int J Biochem Cell Biol 29:1255-1272

66. Ahima RS, Prabakaran D, Mantzoros C et al (1996) Role of leptin in neuroendocrine responses to fasting. Nature 832:25-32

67. Myers MG Jr, Heymsfield SB (2012) Toward a Clinical Definition of Leptin Resistance. Defining clinical leptin resistance Challenges and opportunities. Cell Metab 15:150-156
68. Nindl BC, Kraemer WJ, Arciero PJ et al (2002) Leptin concentrations experience a delayed reduction after resistance exercise in men. Med Sci Sports Exerc 34:608-613

69. Plonka M, Toton-Morys A, Adamski P et al (2011) Association of the physical activity with leptin blood serum level, body mass indices and obesity in schoolgirls. J Physiol Pharmacol 62:647-656

70. Guelfi KJ, Donges CE, Duffield R (2013) Beneficial effects of 12 weeks of aerobic compared with resistance exercise training on perceived appetite in previously sedentary overweight and obese men. Metabolism 62:235-243

71. Gill MS, Toogood AA, Jones J, Clayton PE, Shalet SM (1999) Serum leptin response to the acute and chronic administration of growth hormone $(\mathrm{GH})$ to elderly subjects with $\mathrm{GH}$ deficiency. J Clin Endocrinol Metab 84:1288-1295

72. McClain DA, Alexander T, Cooksey RC, Considine RV (2000) Hexosamines stimulate leptin production in transgenic mice. Endocrinology 141:1999-2002

73. Rebello CJ, Kirwan JP, Greenway FL (2020) Obesity, the most common comorbidity in SARS-CoV-2: is leptin the link? Int J Obes (Lond) 44(9):1810-1817. Doi: https://doi.org/10.1038/ s41366-020-0640-5. Epub 2020 Jul 9. PMID: 32647360; PMCID: PMC7347260

74. Wang J, Xu Y, Zhang X, et al (2021) Leptin correlates with monocytes activation and severe condition in COVID-19 patients. J Leukoc Biol. Doi: https://doi.org/10.1002/JLB.5HI10 20-704R. Epub ahead of print. PMID: 33404078

75. Bircher S, Knechtle B (2004) Relationship between fat oxidation and lactate threshold in athletes and obese women and men. $\mathrm{J}$ Sport Sci Med 3:174-181

76. Parolin ML, Chesley A, Matsos MP, Spriet LL, Jones NL, Heigenhauser GJF (1999) Regulation of skeletal muscle glycogen phosphorylase and PDH during maximal intermittent exercise. Am J Physiol (Endocrinol Metab 40) 277:E890-E900

77. Ekkakis P, Lind E, Vanzou S (2010) Affective responses to increasing levels of exercise intensity in normal weight, overweight, and obese middle-aged women. Obesity (Silver Spring) 18:79-85

78. Lanzi S, Codecasa F, Cornacchia M et al (2015) Short-term HIIT and Fatmax training increase aerobic and metabolic fitness in men with class II and III obesity. Obesity 23:1978-1994

79. Helgerud J, Hoydal K, Wang E et al (2007) Aerobic high-intensity intervals improve $\mathrm{VO}_{2}$ max more than moderate training. Med Sci Sports Exerc 39:665-671

80. Gillen JB, Percival ME, Ludzki A, Tarnopolsky MA, Gibala MJ (2013) Interval training in the fed or fasted state improves body composition and muscle oxidative capacity in overweight women. Obesity (Silver Spring) 21:2249-2255

81. Gibala MJ, Little JP, MacDonald MJ, Hawley JA (2012) Physiological adaptations to low-volume, high-intensity interval training in health and disease. J Physiol 590:1077-1084

82. Gillen JB, Gibala MJ (2014) Is high-intensity interval training a time-efficient exercise strategy to improve health and fitness ? Appl Physiol Nutr Metab 39:409-412

83. Weston KS, Wisloff U, Coombes JS (2014) High-intensity interval training in patients with lifestyle-induced cardio-metabolic disease: a systemic review and meta-analysis. Br J Sports Med 48:1227-1234

84. Astorino TA, Shubert MM, Palumbo E et al (2013) Magnitude and time course of change in maximal oxygen uptake in response to distinct regimens of chronic interval training in sedentary women. Eur J Appl Physiol 113:2361-2369

85. Houmard JA, Tanner CJ, Slentz CA, Duscha BD, McCartney JS, Kraus WE (2004) Effect of the volume and intensity of exercise training on insulin sensitivity. J Appl Physiol 96:101-106 
86. Venables MC, Jeukendrup AE (2008) Endurance training and obesity: effect on substrate metabolism and insulin sensitivity. Med Sci Sports Exerc 40:495-502

87. Bajaj M, Suraamornkul S, Romanelli A et al (2005) Effect of a sustained reduction in plasma free fatty acid concentrations on intramuscular long-chain fatty Acyl-CoAs and insulin action in type 2 diabetic patients. Diabetes 54:3148-3153

88. Lunt H, Draper N, Marshall HC et al (2014) High intensity interval training in a real world setting: a randomized controlled feasibility study in overweight inactive adults, measuring change in maximal oxygen uptake. PLoS ONE 9:e83256

89. American College of Sport Medicine (2000) ACSM's guidelines for exercise testing and prescription, 6th edn. Lippincott Williams \& Wilkins, Baltimore (MD), pp 141-145

90. de Glisezinski I, Moro C, Pillard F et al (2003) Aerobic training improves exercise-induced lipolysis in SCAT and lipid utilization in overweight men. Am J Physiol Endocrinol Metab 285:E984-E990

91. Koppo K, Larrouy D, Marques MA et al (2010) Lipid mobilization in subcutaneous adipose tissue during exercise in lean and obese humans. Roles of insulin and natriuretic peptides. Am J Physiol Endocrinol Metab 299:E258-E265

92. Dengel DR, Pratley RE, Hagberg JM, Rogus EM, Goldberg AP (1996) Distinct effects of aerobic exercise training and weight loss on glucose homeostasis in obese sedentary men. J Appl Physiol 81:318-325

93. Salvadori A, Fanari P, Marzullo A et al (2014) Short bouts of anaerobic exercise increase non-esterified fatty acids release in obesity. Eur J Nutr 53:243-249

94. Salvadori A, Fanari P, Brunani A et al (2015) Leptin level lowers in proportion to the amount of aerobic work after four weeks of training in obesity. Horm Metab Res 47:225-231

95. Donnelly JE, Blair SN, Jakicic JM, Manore MM, Rankin JW, Smith BK (2009) ACSM's position stand. Appropriate physical activity intervention strategies for weight loss and prevention of weight regain for adults. Med Sci Sports Exerc 41:459-471

96. Kiens B (2006) Skeletal muscle lipid metabolism in exercise and insulin resistance. Physiol Rev 86:205-243

97. Oshida Y, Yamanouchi K, Hayamizu S, Sato Y (1989) Longterm mild jogging increases insulin action despite no influence on body mass index or $\mathrm{VO}_{2 \max }$. J Appl Physiol 66:2206-2210

98. Jelleyman C, Yates T, O'Donovan G et al (2015) The effects of high-intensity interval training on glucose regulation and insulin resistance: a meta-analysis. Obes Rev 16:942-961

99. Wang J, Liu R, Hawkins M, Barzilai N, Rossetti L (1998) A nutrient-sensing pathway regulates leptin gene expression in muscle and fat. Nature 393:648-688

100. Zaccaria M, Ermolao A, Brugin E, Bergamin N (2013) Plasma leptin and energy expenditure during prolonged, moderate intensity, treadmill exercise. J Endocrinol Invest 36:396-401

101. Taribagil P, Creer D, Tahir H (2021) Long COVID syndrome. BMJ Case Rep 14(4):e241485. https://doi.org/10.1136/bcr-2020241485 (PMID: 33875508)
102. Nainu F, Abidin RS, Bahar MA et al (2020) SARS-CoV-2 reinfection and implications for vaccine development. Hum Vaccin Immunother 16(12):3061-3073. https://doi.org/10.1080/21645 515.2020.1830683 (PMID: 33393854)

103. Saltin B (1990) Anaerobic capacity: Past, present and prospective. In: Taylor AW, Gollnick PP, Green HJ et al (eds) Biochemistry of exercise VII. IL, Human Kinetics Books, Champaign, pp 387-412

104. Connolly DAJ (2012) The anaerobic threshold: over-valued or under-utilized? A novel concept to enhance lipid optimization ! Curr Opin Clin Nutr Metab Care 15:430-435

105. Stefan N, Birkenfeld AL, Schulze MB (2021) Global pandemics interconnected-obesity, impaired metabolic health and COVID19. Nat Rev Endocrinol 17(3):135-149. https://doi.org/10.1038/ s41574-020-00462-1 (Epub 2021 Jan 21 PMID: 33479538)

106. Rahmati-Ahmadabad S, Hosseini F (2020) Exercise against SARS-CoV-2 (COVID-19): Does workout intensity matter? (A mini review of some indirect evidence related to obesity). Obes Med. 19:100245. Doi: https://doi.org/10.1016/j.obmed. 2020.100245. Epub 2020 Apr 27. PMID: 32342019; PMCID: PMC7184978

107. Kemmler W, Schoene D, Kohl M, von Stengel S (2021) Changes in body composition and cardiometabolic health after detraining in older men with osteosarcopenia: 6-month follow-up of the randomized controlled franconian osteopenia and sarcopenia trial (FrOST) study. Clin Interv Aging 6(16):571-582. https:// doi.org/10.2147/CIA.S299867 (PMID:33854307;PMCID: PMC8039436)

108. Wang M, Baker JS, Quan W, Shen S, Fekete G, Gu Y (2020) A Preventive Role of Exercise Across the Coronavirus 2 (SARSCoV-2) Pandemic. Front Physiol. 11:572718. https://doi.org/ 10.3389/fphys.2020.572718 (PMID: 33013486; PMCID: PMC7506115)

109. Baena Morales S, Tauler Riera P, Aguiló Pons A, García Taibo O (2021) Physical activity recommendations during the COVID19 pandemic: a practical approach for different target groups. Nutr Hosp. 38(1):194-200. https://doi.org/10.20960/nh.03363 (PMID: 33371695)

110. Yang S, Guo B, Ao L, Yang C, Zhang L, Zhou J, Jia P (2020) Obesity and activity patterns before and during COVID-19 lockdown among youths in China. Clin Obes 10(6):e12416. Doi: https://doi.org/10.1111/cob.12416. Epub 2020 Oct 2. PMID: 33009706; PMCID: PMC7646045

Publisher's Note Springer Nature remains neutral with regard to jurisdictional claims in published maps and institutional affiliations. 